\title{
AN ELECTROCHEMICAL INTERFACE FOR INTEGRATED BIOSENSORS
}

\author{
Peter Kim, Neeraj Kohli, Brian Hassler, Nathan Dotson, Andrew Mason, \\ R. Mark Worden, and Robert Ofoli
}

\author{
Michigan State University \\ East Lansing, Michigan, USA \\ kimpeter@egr.msu.edu
}

\begin{abstract}
This paper presents an integrated, protein-based, biosensor that can be scaled to form high-density, multi-analyte sensor arrays physically integrated on a signal conditioning circuit die. A fully scalable, post-CMOS-compatible, threeelectrode interface to biochemical sensors has been developed. A silicon substrate electrode system, consisting of $\mathrm{Ti} / \mathrm{Au}$ working and auxiliary electrodes and a $\mathrm{Ti} / \mathrm{Au} / \mathrm{Ag} / \mathrm{AgCl}$ reference electrode has been adapted to biomimetic sensors. The functional $\mathrm{Ag} / \mathrm{AgCl}$ reference electrode is isolated from the environment using a Nafion cation-exchange membrane to extend operation lifetime. To complete the sensor structure, lipid bilayers have been deposited in passivation layer openings formed over individual working electrodes using a special tethering molecule. Total internal reflection microscopy (TIRFM) studies were done to confirm that a wide range of proteins, such as dehydrogenase enzymes and ion channels, can then be embedded into the lipid bilayers. These results verify the potential to form highly selective recognition elements with direct physical connection to readout electronics on the supporting silicon substrate.
\end{abstract}

\section{INTRODUCTION}

The ability to measure analyte concentrations is essential in disease diagnosis and treatment, as well as in many aspects of chemical and biological research. Protein-based electrochemical biosensors are ideally suited for this purpose, because they provide continuous and accurate measurement of analyte concentrations in an electrical format. Proteins are excellent biological recognition elements (BRE) for biosensors, because they offer great diversity, as well as sensitivity and specificity in their ability to bind, transfer, and/or interact with target analytes. By electrically coupling proteins to an electronic transducer, molecular interactions between the protein and the target analyte can be converted into an electrical signal. Because proteins are of nanometer dimensions, electronic transducers can be miniaturized using semiconductor fabrication methods to construct arrays of miniature biosensors on microelectronics chips that are capable of making many measurements simultaneously.
The ability to construct high-density biosensor arrays is currently limited by a lack of standardized interfaces to couple a wide range of proteins to microelectronics chips that provide high resolution, low-noise signal conditioning. The bilayer lipid membrane (BLM) is the primary electrochemical interface found in nature, and many proteins involved in catalysis, recognition, and analyte transport are found bound to BLMs. Because these proteins frequently lose their activity when removed from the BLM, a biomimetic interface consisting of a synthetic BLM assembled on an electrode could serve as an excellent standardized interface that provides effective electrical communication with the underlying electrode. When combined with reliable reference, working and auxiliary electrodes, integrated electrochemical biosensor arrays can be realized, as illustrated conceptually in Figure 1 . Electrochemical interfaces are particularly useful for amperometric biosensors that measure catalytic activity of redox enzymes. Since these processes can be completed on the surface of a chip, after fabrication of integrated circuits (post-CMOS), a fully integrated microsystem with on-chip instrumentation electronics can be realized.

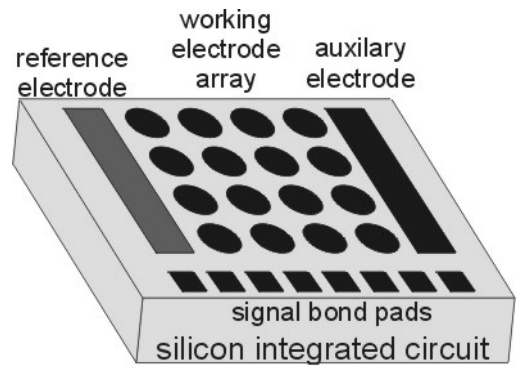

Figure 1: Simplified concept of an electrochemical biosensor array formed on the surface of an integrated circuit chip.

In this paper, ongoing research to construct integrated biosensor array microsystems is discussed. Methods for fabricating reliable, fully scalable, electrochemical electrode arrays on silicon surfaces are presented. Assembly of biomimetic interfaces by depositing lipid bilayers and proteins on electrode surfaces is discussed, and characterization of these interfaces using cyclic 
voltammetry $(\mathrm{CV})$ and total internal reflection fluorescence microscopy (TIRFM) is presented. These results represent the initial steps in the development of high-density, multianalyte biosensor array microsystems.

\section{BIOSENSOR ARRAY MICROSYSTEMS}

There is a clear need for a new sensor platform capable of continuously measuring biochemical analytes with high sensitivity and specificity. Modern technologies provide the potential to realize "biology-to-digital" conversion systems utilizing arrays of miniature sensors with a response time much faster than conventional techniques. These sensor arrays can be combined with complex integrated circuits to form complete microsystems capable of implementing advanced measurement techniques, such as cyclic voltammetry and impedance spectroscopy, which are critical for extracting information from biochemical reactions. There are many distinct advantages to integrating biosensors into miniature microsystems, including compact size, increased sensitivity, and the ability to form high density arrays that allow multiple variables to be measured simultaneously and/or replicates of the same measurement to increase accuracy. These arrays would also provide the unique capability to continuously measure concentration changes of multiple analytes over time, with tremendous advantage in studying the dynamics of chemical and biochemical processes, e.g., disease and metabolic processes. Furthermore, realization of a small, low power, biosensor microsystem platform serves not only traditional applications but also opens many new applications that are not possible with conventional techniques. Figure 2 illustrates the data flow from a test sample to the user, where the key element that must be developed is the integrated biosensor array microsystem. Such a system would be of profound benefit to an extensive range of biological research, biological threat detection and healthcare applications.

\section{BIOLOGICAL RECOGNITION}

Many classes of proteins are being investigated for production of electrochemical biosensors, including enzymes, antibodies, membrane pores and channels, ionophores, and receptors [1,2]. Biosensors using enzymes as the BRE can potentially provide high sensitivities, because the catalytic activity of enzymes allows for much lower detection limits than those obtained with other common binding techniques [1].

The range of analytes measurable using electrochemical biosensors is limited by the almost total absence of commercial biosensors based on dehydrogenase enzymes [3]. Because dehydrogenase enzymes catalyze electrontransfer reactions, their activity can be measured directly with an electrode. However, technical challenges with insitu regeneration of the enzyme's cofactor have hindered commercial development of dehydrogenase-based biosensors [3]. The principal challenge lies in achieving efficient electrical communication between the electrode, cofactor, enzyme, and the electron mediator.

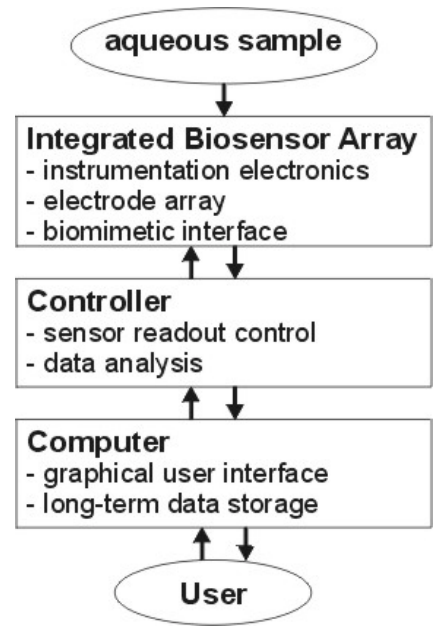

Figure 2: Diagram of a biosensor system which utilizes an integrated biosensor array microsystem as a key interface to biochemical analytes in an aqueous sample.

Ideally, a standard biosensor interface would be suitable for a broad range of BREs. Such an interface would minimize manual processing steps and thus facilitate automation using semiconductor-fabrication methods. The BLM is the basic structure of virtually all cell membranes [4]. Many classes of biological molecules that could serve as BRE are found in nature embedded in BLMs, including enzymes, channel proteins, ion pores, signaling proteins, receptors, and antigens. The microenvironment provided by the BLM is optimal for these molecules, and removing them from the BLM often results in loss of activity. In fact, many molecular processes essential to life, including signal transduction, energy generation, regulation of molecular transport, and maintenance of transmembrane potential gradients require an intact cell membrane [2].

Fortunately, the reconstituting of BREs into artificial BLMs can mimic this optimal natural environment. The resulting biomimetic membranes have been shown to be excellent biosensor interfaces. Biosensor systems based on BLM with associated enzymes, redox mediators, ionophores, ionchannel forming species, antibodies, and DNA are now the subject of extensive research [2,5,6,7].

\section{ELECTROCHEMICAL INTERFACES}

For many BLM-based biosensors, electrochemical interfaces are required to generate electrical signals from biochemical reactions. Well established electrochemical methods, such as cyclic voltammetry, can be used to retrieve data on analyte concentration and reaction kinetics. 
Although these methods are typically employed using bench-top equipment and instruments, in order to realize the biosensor microsystem concept it is necessary to miniaturize the electrochemical system into a microelectronics compatible platform.

A typical electrochemical cell consists of the sample dissolved in a solvent, an ionic electrolyte, and three electrodes; working, counter and reference electrodes. Although two-electrode systems can be utilized, the threeelectrode provides more reliable data, which is especially important as the system is miniaturized and electrical noise becomes more critical. In order to achieve stable and accurate electrochemical measurement, the distance from the reference electrode to the working electrode should be minimized and potential between these two electrodes must be held constant over time. In a conventional threeelectrode system, metal working and auxiliary electrodes are combined with a $\mathrm{Ag} / \mathrm{AgCl}$ reference electrode that is immersed in a saturated $\mathrm{KCl}$ solution to achieve constant electrochemical potential and provide needed $\mathrm{Cl}^{-}$ions. These features must be maintained as the system is miniaturized into an integrated electrochemical interface.

Microelectronics fabrication techniques can be used to produce miniature electrode arrays, and many examples of this are available in the literature. Complete 3-electrode electrochemical cells, including thin film $\mathrm{Ag} / \mathrm{AgCl}$ reference electrodes, have been integrated on silicon chip surfaces [8], and Nafion and polyurethane coatings have been utilized to improve reference electrode stability by providing the needed constant potential and allowing for the flow chlorine ions [9]. Suzuki et. al. have reported improvements to integrated electrochemical cells using complex micromachined structures [10,11]. However, to date, there have been limited efforts to fully integrate electrochemical sensors, including on-chip reference electrodes, monolithically with interface electronics. The fully integrated electrochemical microsystems that have been reported [12,13] are designed for ion selective field effect transistor (ISFET) chemical sensors which have very different characteristics than bioelectrochemical sensors. Furthermore, while both of these microsystems include multiple sensor elements, neither addresses the issues associated with integrating high density electrochemical sensor arrays.

As shown in Figure 3, a four mask process for fabricating a three-electrode configuration on an oxidized silicon substrate has been developed. The integrated electrode system consists of a $\mathrm{Ti} / \mathrm{Au}$ working electrode, a $\mathrm{Ti} / \mathrm{Au}$ auxiliary electrode and a $\mathrm{Ti} / \mathrm{Au} / \mathrm{Ag} / \mathrm{AgCl}$ solid-state reference electrode formed using typical semiconductor fabrication processes. The design utilizes a protective Nafion layer to allow ions to flow from the reference electrode while protecting the electrode from the environment. A final passivation layer isolates the individually exposed electrode surfaces and allows for signal connections at the edge of the electrochemical interface unit. Lipid bilayers can be deposited into the passivation layer opening over the working electrode to complete the biosensor interface. A bilayer tethering molecule, which does not adhere to the passivation layer but bonds well to the Au electrode, can be used to tie the lipid bilayer to the working electrode without fouling the auxiliary or reference electrodes.

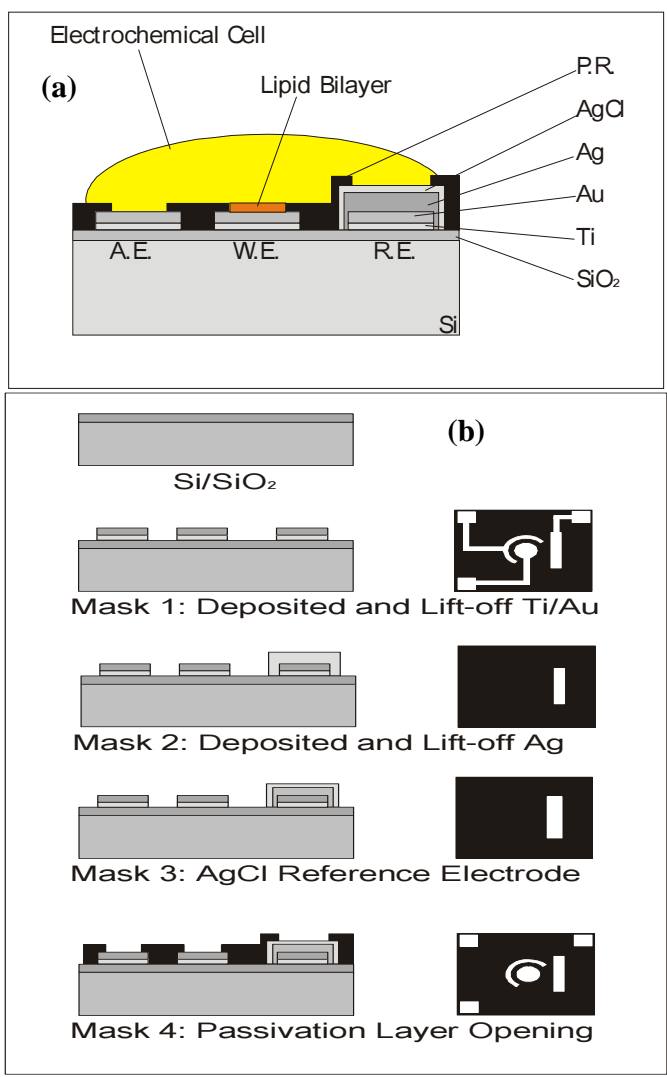

Figure 3: (a) Profile of the three-electrode biosensor system. (b) Process flow.

\section{EXPERIMENTAL RESULTS}

Formation of an integrated biosensor requires 1) fabrication of an integrated three-electrode system, 2) formation of a tethered lipid bilayer on an electrode surface, and 3) adsorption of membrane proteins into an electrode supported lipid bilayers. These three critical elements have been experimentally verified.

\section{Integrated Three-Electrode System}

The integrated three-electrode system discussed in section 4 was fabricated on an oxidized silicon substrate. Photoresist (PR) was deposited and patterned (Mask 1) to expose electrode, routing, and bonding areas. Using $\mathrm{Ti}$ as a substrate adhesive layer, a metal stack of $\mathrm{Ti}(200 \AA) \mathrm{Au}$ $(1500 \AA)$ was then deposited and patterned using a lift-off technique. A new layer of PR was patterned (Mask 2) to 
expose only the reference electrode. A layer of $\mathrm{Ag}(1500 \AA)$ was then deposited and patterned using lift-off. A new PR mask (Mask 3), slightly larger than the reference electrode, was applied and a $\mathrm{Ag} / \mathrm{AgCl}$ electrode was formed by dipping the wafer into $1 \mathrm{M} \mathrm{FeCl}_{3}$ solution for 45 minutes. The wafer was dried in a desiccator for 48 hours to evaporate the solvent and then coated with Nafion (protective polymer layer) to prevent the leakage of chloride ions [9]. The Nafion coated wafer was cured at $120^{\circ} \mathrm{C}$ for 1 hour and stored in a desiccator for 24 hours. To prevent conduction between the electrodes in the presence of an aqueous sample, a final PR mask (Mask 4) was applied and patterned to expose only the electrode and bonding pad areas. The resulting three-electrode chips are shown in Figure 4. These experimental models are large, approximately $15 \mathrm{~mm} \times 15 \mathrm{~mm}$, to simplify subsequent processing and measurement with lipid bilayers.

For initial experiments, a single working electrode was formed (rather than an array) and the working electrode was kept large $(\sim 2 \mathrm{~mm})$ to simplify application of biomimetic membranes. The process can easily be scaled to produce microelectrode arrays of; however methods for depositing biomimetic interfaces on small electrodes remain to be developed.

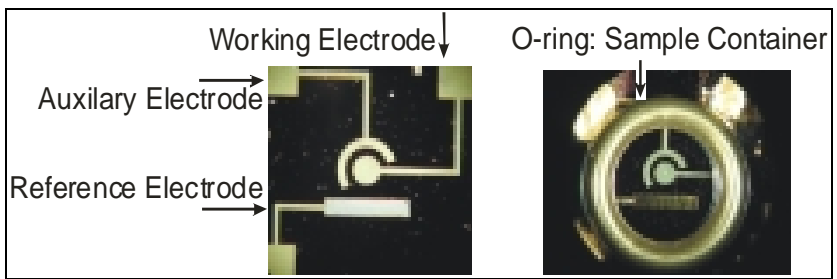

Figure 4: The silicon-based three electrode system (left) with and (right) without a test sample.

To verify the functionality of the integrated three-electrode system shown in Figure 4, cyclic voltammetry experiments were conducted using potassium ferricyanide as the electrolyte. Figure 5 shows the resulting voltammograms at various ferricyanide concentrations. The response is similar to the curve for a conventional electrode system, thus verifying the operation of the integrated electrochemical system.

\section{Formation of a Tethered Lipid Bilayer}

Formation of a tethered bilayer on a gold electrode requires the addition of successive layers between the electrode and the bilayer. The CV curves in Figure 6 show a significant drop in peak current once the final lipid bilayer has been formed (curve ' $c$ '). This demonstrates that a passive lipid bilayer (no active sensor elements) can be properly formed on the gold working electrode and that it adequately reduces current flow to provide a high impedance barrier. Further details of these experiments are given in [14].

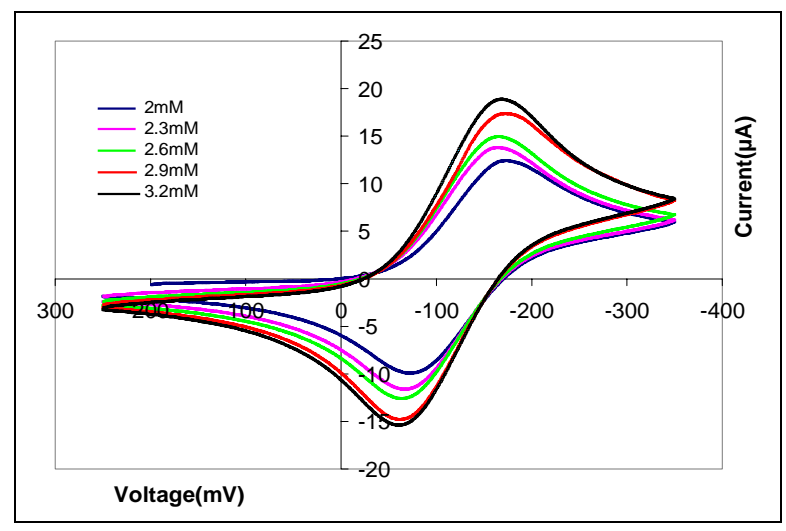

Figure 5: Effect of ferricyanide concentration of cyclic voltammograms obtained using the integrated threeelectrode system.

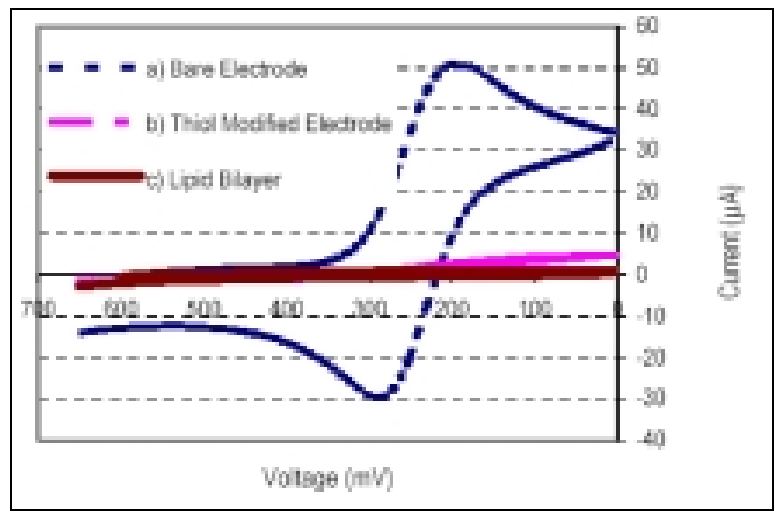

Figure 6: Comparison of cyclic voltammograms for (a) bare gold substrate, (b) thiol modified substrate, and (c) lipid modified substrate.

To determine the temperature stability of a lipid bilayer on a solid electrode support, CV experiments were conducted at different temperatures. The bilayer was first examined by $\mathrm{CV}$ at $25^{\circ} \mathrm{C}$, and it showed a sigmodal shape. The sample was then kept at $\sim 4^{\circ} \mathrm{C}$ for 30 minutes and subsequently reexamined using $\mathrm{CV}$, where is produced the same sigmodal shape. $\mathrm{CV}$ studies were also done at $40^{\circ} \mathrm{C}$ by heating the bilayer electrode module. The lipid bilayer was found to be stable throughout the temperature range investigated. The peak current of the CV curves varied only slightly with temperature, increasing with temperature. This variation may be attributed to the changes in properties such as conductivity of the electrolyte solution. These results suggest that the lipid bilayer is quite stable when subjected to thermal cycling.

\section{Adsorption of Proteins in a Lipid Bilayer}

To confirm that proteins can be embedded into the lipid bilayers, total internal reflection microscopy (TIRFM) studies were done using a biomimetic membrane and a solution of secondary alcohol dehydrogenase (SADH) enzyme. Details of these experiments are presented in [14]. 
The results indicate that the SADH adsorbed strongly onto the lipid bilayer, with no shear induced desorption of the enzyme occurring. For comparison, an SADH adsorption experiment conducted on ITO-coated glass in the absence of a bilayer showed significant protein desorption immediately following exposure of the interface to a protein-free buffer solution at the same flow rate.

\section{CONCLUSIONS}

Techniques for developing integrated electrochemical biosensor arrays have been developed and the key steps have been experimentally verified. Fabrication of a fully functional three-electrode electrochemical interface on the surface of a silicon substrate has been shown. Continuous lipid bilayers have been formed and properly tethered to integrated gold electrodes, and functionality of the bilayers has been confirmed, using CV. TIRFM was used to confirm the adsorption of SADH into lipid bilayers assembled on electrode. Future work will include the optimization of electrode geometries for scaling to high density arrays, development of methods to form bilayers on smaller $(<100 \mu \mathrm{m})$ electrodes, the measurement of protein adsorption kinetics, and the population of integrated biosensors arrays with various membrane proteins.

\section{REFERENCES}

[1] T.Vo-Dinh, and B. Cullum, "Biosensors and biochips: advances in biological and medical diagnostics". Fresenius $J$ Anal Chem., 366 (6-7): pp.540-551, 2000.

[2] M. Trojanowicz. "Miniaturized Biochemical Sensing Devices Based on Planar Bilayer Lipid Membranes", Fresenius J Anal Chem. 371: pp. 246-260, 2001.

[3] M. Prodromidis, and M. Karayannis,. "Enzyme based amperometric biosensors for food analysis". Electroanalysis, 14(4): pp. 241-261, 2001.

[4] H. Tien, A. Ottova-Leitmannova, "Membrane Biophysics: As Viewed from Experimental Bilayer Lipid Membranes", Elsevier, New York, 2000.
[5] P. Krysinki, H. Tien, and A. Ottova. "Charge Transfer Processes and Redox Reactions in Planar Lipid Monolayers and Bilayers." Biotechnol. Prog. 15, pp. 974-990, 1999.

[6] D. Nikolelis, T. Hianik, and U. Krull. "Biosensors based on thin lipid films and liposomes", Electroanalysis, 11: pp. 7-15, 1999.

[7] A. Janshoff, and C. Steinem, "Scanning Force Microscopy of Artificial Membranes", Chembiochem, 2, pp. 798-808, 2001.

[8] K. Yun, H. Kim, S. Joo, J. Kwak and E. Yoon, In situ FTIR Monitoring of Electrochemical Reactions Using a Silicon Micromachined Infrared Thin-layer Cell, Proc. of the International Sensor Conference, Seoul, Korea, pp. 163-164, 2001.

[9] M. Nolan, "Fabrication and Characterization of a Solid State Reference Electrode for Electroanalysis of Natural Waters with Ultramicroelectrodes", Anal. Chem., vol. 69, pp. 12441247, 1997.

[10] H. Suzuki, T. Hirakawa, S. Sasaki, I. Karube, "An integrated three-electrode system with a micromachined liquid-junction $\mathrm{Ag} / \mathrm{AgCl}$ reference electrode," Analytica Chimica Acta 387, pp. 103-112, 1999.

[11] Hiroaki Suzuki,."Advances in the Microfabrication of Electrochemical Sensors and Systems," Electoanalysis, 12 (9), pp. 703-715, 2000.

[12] E. Lauwers, J. Suls, W. Gumbrecht, D. Maes, G. Gielen, and W. Sansen, "A CMOS Multiparameter Biochemical Microsensor With Temperature Control and Signal Interfacing," IEEE J. Solid State Circ., vol. 36, no. 12, pp 2030-2038, December 2001.

[13] T. Tang, E. A. Johannassen, L. Wang, A. Astaras, M. Ahmadian, A. F. Murray, J. M. Cooper, S. P. Beaumont, B. W. Flynn, and D. R. S. Cumming, "Toward a Miniature Wireless Integrated Multisensor Microsystem for Industrial and Biomedical Applications," IEEE Sensors Journal, vol. 2, no. 6, pp. 628-635, December 2002.

[14] N. Kohli, B. Hassler, S. Vaidya, L. Parthasarathy, R. Ofoli, R. M.Worden, P. Kim, and A. Mason "Biomimetic Interface for Integrated Biosensor Arrays," (to appear) Proc. American Inst. Chemical Engineers Annual Meeting, San Francisco, November 2003. 